\title{
Presença de Substâncias consideradas \\ -.-.--- doping em suplementos alimentares -..--.
}

\author{
Presence of substances considered doping in food supplements
}

\section{Resumo}

O uso de substancias que aumentam rendimento é tão antigo quanto as práticas esportivas. Entretanto más escolhas alimentares não podem ser revertidas pela ingestão de suplementos alimentares, que está associada à prática esportiva. Entende-se como doping a verificação de violações das normas do Código Mundial Antidoping proposto pela Agência Mundial Antidopagem (WADA), que demonstrou preocupação quanto ao uso desses produtos, visto que sua fabricação não é regulamentada em diversos países, podendo conter substâncias não declaradas nos rótulos e até mesmo consideradas doping. Este estudo trata-se de uma revisão de literatura para melhor compreender a presença de substâncias dopantes em suplementos alimentares e as medidas para evitá-las. Para localizar as referências bibliográficas utilizadas, foram realizadas pesquisas na base de dados PUBMED/MEDLINE empregando as seguintes palavras-chave: "doping in supplements", "doping", "doping in sports", "supplements", "anti-doping". Múltiplos suplementos demonstram eficácia e segurança em seu uso; por outro lado, muitos não proporcionam as mesmas propriedades e, além de ineficazes, podem conter substâncias consideradas doping pela WADA. Alguns produtos podem conter doses excessivas dessas substâncias e ocorrências de óbito pelo uso de produtos contendo-as já foram evidenciadas. Diferentes estudos demonstram a presença de substâncias dopantes em suplementos, podendo chegar a $58 \%$ das amostras com contaminação. O uso desses produtos deve ser feito com cautela em razão da possibilidade de lesão à saúde e ocorrência de doping. Suplementos alimentares podem conter substâncias consideradas doping pela WADA e devem ser prescritos exclusivamente por nutricionistas e/ou médicos com conhecimento adequado para direcionar, quando necessário, o atleta ao uso. O nível de evidência para eficácia e segurança deve ser considerado e deve-se optar por marcas autênticas quanto aos ingredientes do produto. As legislações que regulamentam a produção e importação desses deve ser mais rigorosa a fim de que casos relacionados ao doping por contaminação deixem de acontecer.

Palavras-chave: Doping em suplementos, doping, doping em esportes, suplementos, anti-doping.

\section{Abstract} The use of performance-enhancing substances is as old as sporting practices. However poor dietary choices can t be reversed by ingestion of dietary supplements, which is associated with sports practice. It is understood as doping the verification of violations of the standards of the World Anti-Doping Code proposed by the World Anti-Doping Agency (WADA) that has expressed concern about the use of those products, since their manufacture is not regulated in several countries and may contain undeclared substances on labels, and even doping substances. This study is a literature review to better understand the doping substance presence in food supplements and measures to avoid them. In order to locate the bibliographic references used, searches were done in the PUBMED/MEDLINE database, using the following keywords: "doping in supplements" "doping" "doping in sports" "supplements" "anti-doping". Multiple supplements demonstrate efficacy and safety in their use; on the other hand, many don't provide the same properties and, in addition to being ineffective, may contain substances considered as doping by WADA. Some products may contain excessive doses of those substances and occurrences of death by the use of products containing them have already been evidenced. Different studies show the presence of dopant substances in supplements, reaching $58 \%$ of the samples with contamination. The use of those products should be done carefully due to the possibility of health damage and doping occurrence. Food supplements may contain substances considered doping by WADA and should be prescribed exclusively by nutritionists and/or physicians with appropriate knowledge to direct, when necessary, the athlete to use. The level of evidence for efficacy and safety should be considered and authentic brands should be chosen. The legislation that regulate the production and importation of such products should be more rigorous so that cases related to doping through contamination no longer occur.

Keywords: Doping in supplements, doping, doping in sports, supplements, anti-doping. 


\section{Introdução}

Atletas movidos pelo sonho de se tornarem os mais rápidos, mais altos e mais fortes (Citius, Altius, Fortius), buscam a vitória independentemente do custo ${ }^{1}$. Até mesmo quando isso significa a utilização de substâncias e métodos banidos pelas organizações antidoping.

Após os eventos que abalaram o mundo do ciclismo no verão de 1998, o Comitê Olímpico Internacional (COI) decidiu convocar a Conferência Mundial sobre Doping, reunindo todas as partes envolvidas na luta contra o doping $^{2}$.

\section{A Agência Mundial Antidopagem (WADA)}

foi estabelecida em 1999 como uma agência internacional independente composta e financiada igualmente pelo movimento esportivo e pelos governos do mundo. Suas principais atividades incluem pesquisa científica, educação, desenvolvimento de capacidades antidoping e monitoramento do Código Mundial Antidoping, o documento que harmoniza as políticas antidoping em todos os esportes e em todos os países ${ }^{3}$.

Entende-se como doping a verificação de uma ou mais violações das normas propostas pelo Código Mundial Antidoping da WADA4, descritas no Quadro 1.

\section{Quadro 1. Normas do Código Mundial Antidopage}

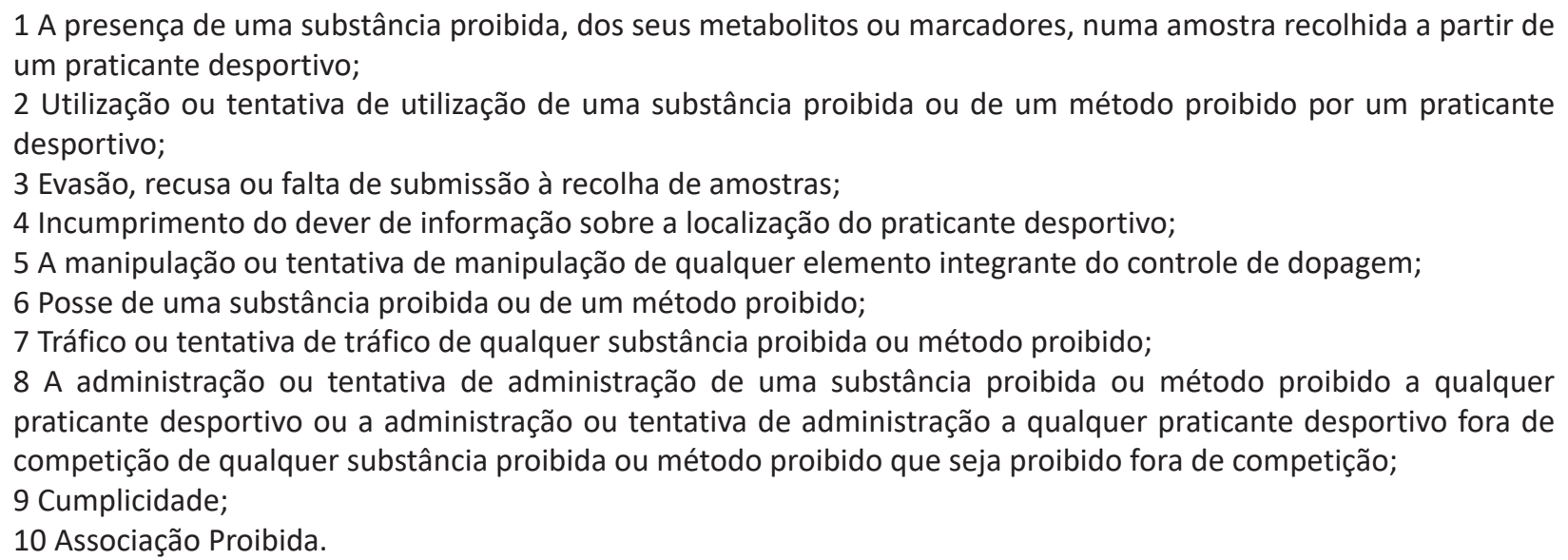

Fonte: Adaptado de AMA-WADA, (2015).

Quadro 2. Lista Proibida da Agência Mundial Antidoping (WADA), válida de 1 de janeiro de 2019 até 31 de dezembro de 2019.

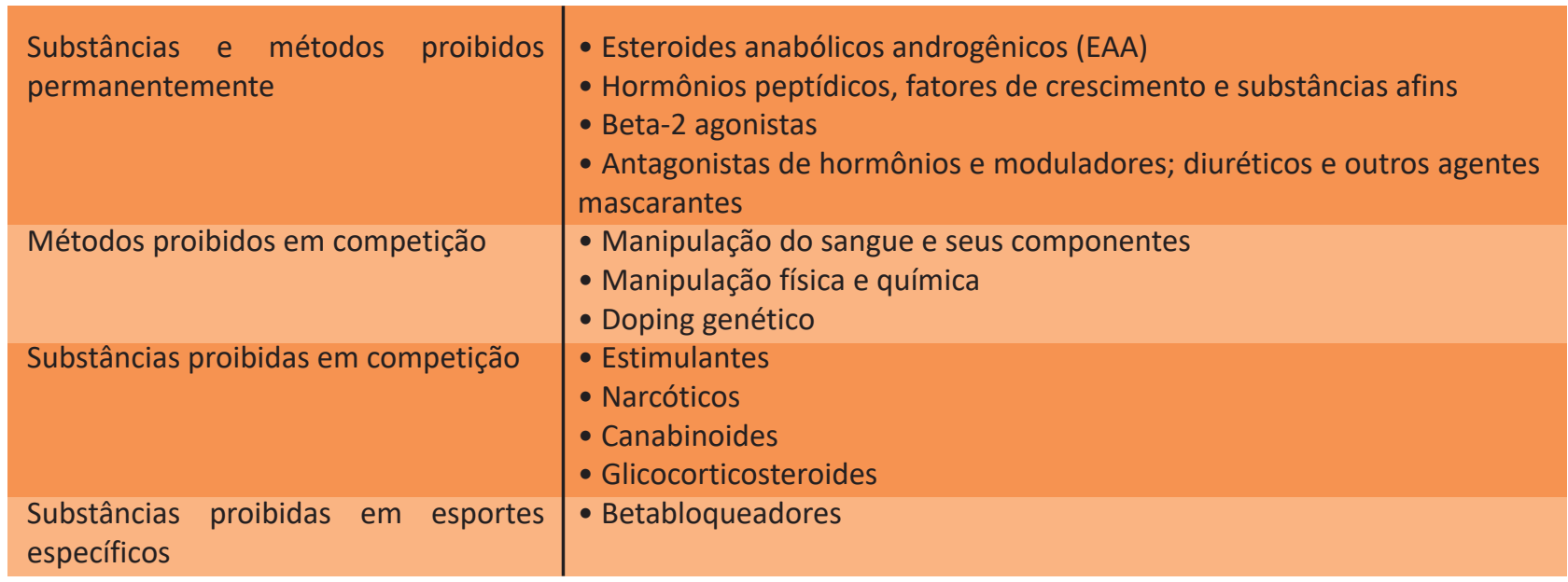

Fonte: Adaptado de WADA (2019). 
A primeira lista de substâncias e métodos proibidos foi publicada em 1963 pelo Comitê Olímpico Internacional (COI), entidade responsável por sua criação. Hoje em dia, essa lista é publicada anualmente pela WADA em um documento denominado "Lista proibida".

A Organização cita os seguintes esportes que se enquadram como "esportes específicos" nos quais os betabloqueadores são proibidos: arco e flecha; automobilismo; bilhar em todas as modalidades; dardos; golf; tiro; esqui/ snowboard em saltos de esqui, freestyle aerials/ halfpipe snowboard halfpipe/big air; esportes subaquáticos em apneia de peso constante com ou sem nadadeiras, apneia dinâmica com e sem nadadeiras, apneia por imersão livre, apneia do tipo jump blue, caça submarina, apneia estática, tiro ao alvo e apneia por peso variável ${ }^{5}$.

$\mathrm{O}$ uso de substâncias que buscam o aumento do rendimento físico é quase tão antigo quanto o desenvolvimento das atividades físicas organizadas $^{6}$. O consumo de suplementos alimentares foi considerado fator associado à prática de esportes individuais e de altas horas de treinamento ${ }^{7}$. Desde o uso de pílulas multivitamínicas utilizadas com a crença de medida de seguro, até os mais exóticos suplementos encontrados nas prateleiras de lojas de alimentos saudáveis ${ }^{8}$.

Más escolhas alimentares não podem ser revertidas pela ingestão de suplementos alimentares, entretanto uma dieta variada e corretamente embasada pode maximizar os benefícios que suplementação pode fornecer ${ }^{9}$.

A preocupação sobre o uso de suplementos foi evidenciada pela WADA ${ }^{10}$, visto que vários países não regulamentam a fabricação desses, tornando mais fácil que ingredientes apresentados nos rótulos não correspondam fielmente aos presentes nos suplementos e, em alguns casos, até mesmo substâncias proibidas pela regulamentação antidoping podem estar presentes.

Este estudo trata-se de uma revisão de literatura para melhor compreender a presença de substâncias dopantes em suplementos alimentares e as medidas para evitá-las. Para localizar as referências bibliográficas utilizadas neste trabalho, foram realizadas pesquisas nas seguintes bases de dados: PUBMED/MEDLINE e Google Acadêmico, empregando as seguintes palavras-chave: "doping in supplements", "doping", "doping in sports", "supplements" e "anti-doping".

\section{Resultados e Discussão}

Forte et al. ${ }^{11}$ relatam um caso de infarto do miocárdio em atleta jovem que foi associado ao uso de um suplemento dietético rico em efedrina. Nos dias de hoje, a substância já é considerada doping $^{12}$.

Em um estudo publicado em 2006, Baume et al. ${ }^{13}$ observaram que um em cinco suplementos à venda estava contaminado, acidentalmente ou intencionalmente, com substâncias não declaradas nos rótulos.

Além disso, em uma revisão de literatura conduzida por Parra, Palma e Pierucci ${ }^{14}$ observouse que algumas investigações detectaram doses excessivas de ingredientes potencialmente tóxicos não indicados no rótulo e considerados doping pela WADA. Entre as substâncias proibidas encontradas estavam os esteroides anabólico-androgênicos e os psicoestimulantes.

Revisando 23 estudos que continham, em sua maioria, a proposta de identificar substâncias consideradas doping em suplementos alimentares, Martínez-Sanz et al. ${ }^{15}$ identificaram uma variação entre 12 e $58 \%$ de valores de contaminação.

Geyer et al. ${ }^{16}$, em 14,8 \% dos 634 suplementos analisados, observaram a presença de próhormôniosnãodeclarados nos rótulos. JáMarkman et al. ${ }^{17}$ analisaram 111 amostras de suplementos e puderam identificar aproximadamente $25 \%$ destes contendo substâncias esteroidais. Dentre elas, decanoato de testosterona, propionato de testosterona, isocaproato de testosterona, e outras substâncias esteroidais não identificadas.

Instituições como a American Dietetic Association, Dietitians of Canada e American College of Sports Medicine (ACSM) ${ }^{18}$ e Juhn ${ }^{19}$ já sugeriram que o uso de suplementos alimentares por praticantes de atividades físico-esportivas deveria ser feito com cautela e somente após cuidadosa avaliação, uma vez que, além dos possíveis efeitos favoráveis defendidos na 
literatura, o uso de suplementos dietéticos tem, ao mesmo tempo, potencial para causar danos e efeitos adversos ${ }^{18-20}$, visto que diversos produtos estão contaminados com a presença de anabolizantes e estimulantes ${ }^{21}$, prejudicando a saúde, o desempenho, a subsistência do atleta e sua reputação se por ventura uma violação das normas antidopagem ocorrer ${ }^{22}$.
O treinador e o nutricionista são uma importante fonte de informação dos atletas. Portanto tornase fundamental que esses profissionais tenham conhecimentos adequados que lhes permitam informar e aconselhar os atletas de forma $\operatorname{correta}^{23}$, baseando-se em evidências para que seus direcionamentos para os atletas sejam feitos de maneira inteligente e cuidadosa.

Quadro 3. Resumo dos mais comuns suplementos alimentares e nível de evidência e segurança.

\begin{tabular}{|c|c|c|c|c|}
\hline Autor & & $\begin{array}{c}\text { Forte evidência para } \\
\text { comprovar eficácia e } \\
\text { segurança }\end{array}$ & $\begin{array}{l}\text { Evidências limitadas para } \\
\text { comprovar eficiência }\end{array}$ & $\begin{array}{l}\text { Origem Pouca evidência para } \\
\text { comprovar eficácia e segurança }\end{array}$ \\
\hline \multirow[t]{3}{*}{ Close et al. ${ }^{24}$} & Endurance & $\begin{array}{c}\text { Cafeína } \\
\text { Géis/bebidas de } \\
\text { carboidrato } \\
\text { Beta-alanina } \\
\text { Suco de beterraba } \\
\text { Bicarbonato de sódio/ } \\
\text { Citrato } \\
\text { Antioxidantes }\end{array}$ & $\begin{array}{c}\text { Taurina } \\
\text { Cherry Active } \\
\text { L-Carnitina }\end{array}$ & $\begin{array}{c}\text { Efedrina } \\
\text { Metilhexanamina } \\
\text { Suplementos herbais } \\
\text { Citrulina malato } \\
\text { L-Arginina } \\
\text { Sinefrina }\end{array}$ \\
\hline & Força/Tamanho & $\begin{array}{l}\text { Creatina } \\
\text { Proteína }\end{array}$ & $\begin{array}{l}\text { Leucina } \\
\text { BCAAs }\end{array}$ & $\begin{array}{c}\text { Zinco Magnésio Aspartato (ZMA) } \\
\text { Qualquer "Anabólico" } \\
\text { Testosterona } \\
\text { Intensificadores } \\
\text { Suplementos herbais } \\
\text { Colostro }\end{array}$ \\
\hline & Saúde & $\begin{array}{l}\text { Probióticos } \\
\text { Eletrólitos } \\
\text { Vitamina D }\end{array}$ & $\begin{array}{l}\text { Vitamina C } \\
\text { Multivitamínico } \\
\text { Glucosamina } \\
\text { Quercetina } \\
\text { Glutamina } \\
\text { Óleo de peixe } \\
\text { Colágeno }\end{array}$ & $\begin{array}{c}\text { Magnésio } \\
\text { Suplementos herbais }\end{array}$ \\
\hline $\begin{array}{l}\text { Kerksick et } \\
\text { al. }^{25}\end{array}$ & $\begin{array}{l}\text { Crescimento } \\
\text { muscular }\end{array}$ & $\begin{array}{l}\text { HMB (Beta-hidroxi-beta- } \\
\text { metilbutirato) } \\
\text { Creatina monohidratada } \\
\text { Aminoácidos essenciais } \\
\text { Proteína }\end{array}$ & \begin{tabular}{|c|} 
Adenosina trifosfato \\
Aminoácidos de cadeia ramificada \\
(BCAAs) \\
Ácido fosfatídico
\end{tabular} & $\begin{array}{c}\text { Sulfato de agmatina } \\
\text { Alfa-cetoglutarato } \\
\text { Arginina } \\
\text { Boro } \\
\text { Cromo } \\
\text { Ácido linoleico conjugado } \\
\text { Ácido d-aspártico } \\
\text { Beta Ecdisterona } \\
\text { Extrato de feno-grego } \\
\text { Gama Orizanol (Ácido ferúlico) } \\
\text { Glutamina } \\
\text { Peptídeos liberadores de } \\
\text { hormônio de crescimento e } \\
\text { Secretagogos } \\
\text { Isoflavonas }\end{array}$ \\
\hline
\end{tabular}




\begin{tabular}{|c|c|c|c|c|}
\hline Autor & & $\begin{array}{c}\text { Forte evidência para } \\
\text { comprovar eficácia e } \\
\text { segurança }\end{array}$ & $\begin{array}{l}\text { Evidências limitadas para } \\
\text { comprovar eficiência }\end{array}$ & $\begin{array}{l}\text { Origem Pouca evidência para } \\
\text { comprovar eficácia e segurança }\end{array}$ \\
\hline & & & & $\begin{array}{c}\text { Gama Orizanol (Ácido ferúlico) } \\
\text { Glutamina } \\
\text { Peptídeos liberadores de } \\
\text { hormônio de crescimento e } \\
\text { Secretagogos } \\
\text { Isoflavonas } \\
\text { Ornitina alfa cetoglutarato (OKG) } \\
\text { Pró-hormônios } \\
\text { Polissacarídeos sulfatados } \\
\text { Tribulus terrestris } \\
\text { Sulfato de vanádio } \\
\text { ZMA }\end{array}$ \\
\hline & $\begin{array}{l}\text { Melhora de } \\
\text { performance }\end{array}$ & \begin{tabular}{|c|} 
Beta-alanina \\
Cafeína \\
Carboidrato \\
Creatina monohidratada \\
Bicarbonato de sódio \\
Fosfato de sódio \\
Água e bebidas esportivas
\end{tabular} & \begin{tabular}{|c|} 
L-Alanil-L-Glutamato \\
Ácido araquidônico \\
Aminoácidos de cadeia ramificada \\
(BCAAs) \\
Citrulina \\
Aminoácidos essenciais \\
Glicerol \\
HMB (Beta-hidroxi-beta- \\
metilbutirato) \\
Nitratos \\
Carboidratos e proteínas pós- \\
exercício \\
Quercetina
\end{tabular} & $\begin{array}{c}\text { Arginina } \\
\text { Carnitina } \\
\text { Glutamina } \\
\text { Inosina } \\
\text { Triglicerídeos de cadeia média } \\
\text { (TCM) } \\
\text { Ribose }\end{array}$ \\
\hline
\end{tabular}

Nutricionistas devem se aprofundar para melhor orientar atletas quanto à possibilidade da presença de substâncias consideradas doping em suplementos alimentares ${ }^{26}$. E com o intuito de minimizar o risco de contaminação, quando optarem pelo uso, atletas devem comprar apenas suplementos nutricionais de empresas que realizem controle de qualidade para evitar pró-hormônios e/ou possam garantir que seus produtos não têm nenhum contato com pró-hormônios, tanto no processo de produção quanto no transporte ${ }^{16}$.

\section{Conclusão}

Suplementos alimentares podem conter substâncias potencialmente tóxicas, inclusive consideradas doping pela WADA, e devem ser prescritos exclusivamente por nutricionistas ou médicos que possuam o conhecimento necessário para direcionar o atleta ao uso, somente quando se mostrar necessário. Deve-se considerar o nível de evidência da eficácia e principalmente da segurança do produto, buscando por marcas que apresentem autenticidade quanto aos ingredientes. Evidencia-se que as legislações que regulamentam a produção e importação de suplementos deveria exigir um controle mais rigoroso, a fim de que casos relacionados ao doping por contaminação deixem de acontecer.

\section{Referências bibliográficas}

1. BIRZNIECE, V. Doping in sport: effects, harm and misconceptions. Internal medicine journal, 45 (3): 239-248, 2015.

2. HANSTAD, D.V. Drug scandal and organizational change within the International Ski Federation: A figurational approach. European sport management quarterly, 8 (4): 379-398, 2008.

3. AGÊNCIA MUNDIAL ANTIDOPING. In: Wikipédia. Disponível em: https://pt.wikipedia.org/wiki/Ag\%C3\%AAncia_Mundial_Antidopingo. Acesso em: 01/11/2018. 
4. AGÊNCIA MUNDIAL ANTIDOPING. Código Mundial Antidopagem. Disponível em: https://www.wada-ama.org/sites/default/files/ resources/files/codigo_mundial_antidopagem_2015.pdf. Acesso em: 08/10/2018.

5. AGÊNCIA MUNDIAL ANTIDOPING. Lista proibida 2019. Disponível em: https://www.wada-ama.org/sites/default/files/wada_2019_ english_prohibited_list.pdf. Acesso em: 08/10/2018.

6. TAVARES, O. Doping argumentos em discussão. Movimento; 8 (1): 41-55, 2002.

7. NABUCO, H.C.G.; RODRIGUES, V.B.; RAVAGNANI, C.F.C. Fatores associados ao uso de suplementos alimentares entre atletas: revisão sistemática. Revista Brasileira de Medicina do Esporte; 22 (5): 412-419, 2016.

8. MAUGHAN, R.J. Contamination of dietary supplements and positive drug tests in sport. Journal of sports sciences; 23 (9): $883-889$, 2005.

9. LARSON-MEYER, D.E.; WOOLF, K.; BURKE, L. Assessment of nutrient status in athletes and the need for supplementation. International journal of sport nutrition and exercise metabolism; 28 (2): 139-158, 2018.

10. WORLD ANTI-DOPING AGENCY. Athlete guide. Montreal: World Anti-Doping Agency, 2005.

11. FORTE, R. Y.; PRECOMA-NETO, D.; NETO, N. C. et al. Infarto do miocárdio em atleta jovem associado ao uso de suplemento dietético rico em efedrina. Arquivos Brasileiros de Cardiologia; 87 (5): 179-181, 2006.

12. AGÊNCIA MUNDIAL ANTIDOPING. Lista proibida 2007. Disponível em: https://www.wada-ama.org/sites/default/files/resources/ files/WADA_Prohibited_List_2007_EN.pdf. Acesso em: 06/11/2018.

13. BAUME, N.; MAHLER, N.; KAMBER, M. et al. Research of stimulants and anabolic steroids in dietary supplements. Scandinavian journal of medicine \& science in sports; 16 (1): 41-48, 2006.

14. PARRA, R.M.T.; PALMA, A.; PIERUCCI, A.P.T.R. Contaminação de suplementos dietéticos usados para prática esportiva: uma revisão de literatura. Revista Brasileira de Ciências do Esporte; 33 (4): 1071-1084, 2011.

15. MARTÍNEZ-SANZ, J.; SOSPEDRA, I.; ORTIZ, C. et al. Intended or unintended doping? A review of the presence of doping substances in dietary supplements used in sports. Nutrients; 9 (10): 1093, 2017.

16. GEYER, H.; PARR, M. K.; MARECK, U. et al. Analysis of non-hormonal nutritional supplements for anabolic-androgenic steroids-results of an international study. International journal of sports medicine; 25 (02): 124-129, 2004.

17. MARKMAN, B.E.O.; KOSCHTSCHAK, M.R.W.; UESSUGUI, O. et al. Pesquisa e identificação de anabolizantes em alimentos elaborados para praticantes de atividades físicas, suplementos vitamínicos e ou minerais. Boletim Epidemiológico Paulista; 5 (60): 4-8, 2008.

18. AMERICAN DIETETIC ASSOCIATION, DIETITIANS OF CANADA AND AMERICAN COLLEGE OF SPORTS MEDICINE. Position of American Dietetic Association, Dietitians of Canada and American College of Sports Medicine: nutrition and athletic performance. Journal of the American Dietetic Association; 100 (12): 1543-56, 2000.

19. JUHN, M.S. Popular sports supplements and ergogenic aids. Sports Medicine; 33 (12): 921-939, 2003.

20. ZOVICO, P.V.C.; CAVATTI, R.M.; CURTY, V.M. et al. Suplementos contendo DMAA: mitos e verdades. Revista Brasileira de Nutrição Esportiva; 12 (72): 443-462. 2018.

21. VAN THUYNE, W.; VAN EENOO, P.; DELBEKE, F.T. Nutritional supplements: prevalence of use and contamination with doping agents. Nutrition research reviews; 19 (1): 147-158, 2006.

22. MAUGHAN, R.J. IOC Medical and Scientific Commission reviews its position on the use of dietary supplements by elite athletes. British Journal of Sports Medicine; 52 (1): 418-419, 2018.

23. FERNANDES, M.J.A. Uso de suplementos nutricionais por atletas das seleções nacionais masculinas portuguesas. Dissertação (mestrado) - Universidade do Porto. Porto, 2009.

24. CLOSE, G.L.; HAMILTON, D.L.; PHILP, A. et al. New strategies in sport nutrition to increase exercise performance. Free Radical Biology and Medicine; 98 (1): 144-158, 2016.

25. KERKSICK, C.M.; WILBORN, C.D.; ROBERTS, M.D. et al. ISSN exercise \& sports nutrition review update: research \& recommendations. Journal of the International Society of Sports Nutrition; 15 (1): 38, 2018.

26. CASTANHO, G.K.F.; FONTES, E.B.; FERNANDES, P.T. O perigo da contaminação de suplementos alimentares com substâncias ilícitas para os praticantes de exercício físico e esporte. Revista da Faculdade de Educação Física da UNICAMP; 12 (1): 161-180, 2014. 